\title{
NUMERICAL METHODS FOR COMPUTING NONLINEAR EIGENPAIRS: PART I. ISO-HOMOGENEOUS CASES*
}

\author{
XUDONG $\mathrm{YAO}^{\dagger}$ AND JIANXIN ZHOU ${ }^{\ddagger}$
}

\begin{abstract}
With a Rayleigh quotient formulation, a local minimax method is developed to solve a class of (iso-homogeneous) nonlinear eigenpair problems for multiple solutions in Banach spaces following their instability order. The algorithm is implemented to compute (weighted) eigenpairs of the p-Laplacian. Numerical eigenfunctions are illustrated by their graphics. Several interesting phenomena have been observed and are open for further investigation. Mathematical analysis related to convergence and instability order of computed eigenfunctions, etc., is also presented.
\end{abstract}

Key words. nonlinear eigenpairs, multiple critical points, local minimax method

AMS subject classifications. 45R50, 35P30, 58E05, 35J65

DOI. $10.1137 / 060651859$

1. Introduction and some preliminaries. The semilinear Schrödinger equation

$$
i w_{t}+\Delta w+\kappa f(|w|) w=0
$$

where $\kappa$ is a physical parameter, is one of the canonical nonlinear equations in physics, arising in various fields, such as nonlinear optics, plasma fluids, quantum physics, Bose-Einstein condensates, and surface waves. For example, the Gross-Pitaevskii equation $[10,27]$

$$
\begin{aligned}
i \psi_{t} & =-\frac{1}{2} \Delta \psi+V(x) \psi+\beta|\psi|^{2} \psi, \quad t>0, x \in \Omega \subseteq \mathbb{R}^{d}, \\
\psi(x, t) & =0, \quad x \in \Gamma=\partial \Omega, t \geq 0,
\end{aligned}
$$

on a bounded open domain $\Omega \in \mathbb{R}^{d}$, is extensively used to model the single particle of the Bose-Einstein condensate after the first experimental realization of the BoseEinstein condensate in dilute weakly interacting gases shows great agreement. To study stability/instability, pattern, and other solution properties, people are interested in finding the standing (solitary) wave solutions of the form $w(x, t)=u(x) e^{-i \lambda t}$, where $\lambda$ is a normalized potential and $u$ is a real function of $x$, and also the steady-state solutions $w(x, t)=u(x)$. Then (1.1) leads to two semilinear elliptic PDEs

$$
\begin{aligned}
& \lambda u(x)+\Delta u(x)+\kappa f(|u|) u(x)=0, \\
& -\Delta u(x)=\kappa f(|u|) u(x) .
\end{aligned}
$$

Several different types of quasi-linear Schrodinger equations are also proposed to model "Schrodinger flows" in various fields; see [16, 14, 22] for general study and

*Received by the editors February 9, 2006; accepted for publication (in revised form) December 20, 2006; published electronically June 12, 2007. This research was supported in part by NSF grant DMS-0311905.

http://www.siam.org/journals/sisc/29-4/65185.html

${ }^{\dagger}$ Department of Mathematics, University of Connecticut, Storrs, CT 06269 (xudong@math.uconn. edu).

${ }^{\ddagger}$ Department of Mathematics, Texas A\&M University, College Station, TX 77843 (jzhou@math. tamu.edu). 
physical applications of this topic. When non-Darcian or non-Newtonian fluids are considered, Darcy's law is replaced by other relations between the specific discharge (shear stress) $\vec{q}$ and the flow head $w$. One of them is the Izbash formula $[15,4]$ : $\vec{q}=-M|\nabla w|^{p-2} \nabla w$ for some constant $M>0$ and the (rheological) characteristic $p>1$ of the fluid. Then after replacing the Laplacian $\Delta u$ by the p-Laplacian $\Delta_{p} u(x)=$ $\nabla \cdot\left(|\nabla u(x)|^{p-2} \nabla u(x)\right),(1.3)$ and (1.4) are generalized to

$$
\begin{aligned}
& \lambda u(x)+\Delta_{p} u(x)+\kappa f(|u(x)|) u(x)=0, \\
& -\Delta_{p} u(x)=\kappa f(|u(x)|) u(x),
\end{aligned}
$$

two quasi-linear elliptic PDEs. When $p=2, p<2, p>2$, the fluid is called, respectively, (Darcian) Newtonian, pseudoplastic, dilatant. The p-Laplacian $\Delta_{p}$ is also used to model phenomena in reaction-diffusion in porous media $\left(p=\frac{3}{2}\right)$, nonlinear elasticity $(p>2)$, and glaciology $\left(p \in\left(1, \frac{4}{3}\right)\right)$; see $[4,24]$ and references there. When $p=2$, (1.5) and (1.6) reduce to (1.3) and (1.4). When the nonlinear term $f(|u(x)|) u(x)$ is primitive, i.e., $f(|t|) t=\frac{d}{d t} F(t)$ for some function $F$, the problems are variational and (1.5) and (1.6) are, respectively, the Euler-Lagrange equations $\left(J^{\prime}(\cdot)=0\right)$ of the (energy) functionals

$$
\begin{aligned}
J(u, \lambda) & =\int_{\Omega}\left[\frac{1}{p}|\nabla u(x)|^{p}-\kappa F(u(x))-\frac{\lambda}{2}\left(|u(x)|^{2}-\alpha\right)\right] d x, \\
J(u) & =\int_{\Omega}\left[\frac{1}{p}|\nabla u(x)|^{p}-\kappa F(u(x))\right] d x .
\end{aligned}
$$

People are interested in knowing the values of $\lambda$ and $\kappa$ for which (1.5) and (1.6) have nontrivial, positive, or nodal solutions. It turns out that those values are either directly determined by or closely related to the eigenvalues of $\Delta_{p}$ as defined in (1.9) [23]. So we leave more general problems (1.5) and (1.6) to a subsequent paper [38] and consider, in this paper, computing eigenpairs $(\lambda, u) \in \mathbb{R} \times(B \backslash\{0\})$ of the (weighted) p-Laplacian

$$
-\Delta_{p} u(x)=\lambda w(x)|u(x)|^{p-2} u(x) \quad \forall x \in \Omega, \quad u \in B=W_{0}^{1, p}(\Omega),
$$

where $\Omega \subset \mathbb{R}^{n}$ is open and bounded, $w: \Omega \rightarrow \mathbb{R}$ is a weight function, and $|\nabla u|^{2}=$ $\sum_{i=1}^{n}\left(\frac{\partial u}{\partial x_{i}}\right)^{2}$. Equation (1.9) is also closely related to the resonance phenomenon, spectral theory of nonlinear operators, Sobolev embedding and inequality, etc.; see, e.g., $[7,24]$. When $p=2,(1.9)$ reduces to an eigenproblem of the Laplacian $\Delta$ and is well known for its importance in many applications and theoretical studies. Thus (1.9) provides a new way to study the eigenproblem of $\Delta$ by embedding it into a one-parameter family of operators $\Delta_{p}$. Hence new and better understanding can be obtained; see Figure 15 and its remarks. For the existence of multiple solutions to $(1.9)$, we refer to $[18,3]$.

Equation (1.9) leads us to study the following nonlinear eigenpair problem (NEP). Let $B$ be a Banach space, $B^{\prime}$ its dual, $\langle$,$\rangle the dual relation, and \|\cdot\|$ the norm in $B$. Let $F^{\prime}$ and $G^{\prime}$ be the Fréchet derivatives of functionals $F$ and $G$ in $C^{1}(B, \mathbb{R})$. For given $\alpha>0$, find eigenpairs $(\lambda, u) \in \mathbb{R} \times(B \backslash\{0\})$ such that (s.t.)

$$
F^{\prime}(u)=\lambda G^{\prime}(u) \text { in } B^{\prime} \quad \text { subject to } G(u)=\alpha,
$$

where $\lambda$ is called an eigenvalue and $u$ is called an eigenfunction associated with $\lambda$. Under certain conditions, existence of countable eigenpairs of (1.10) can be established (see Proposition 44.26 in [39]). 
Recall that for $J \in C^{1}(B, \mathbb{R})$, a point $u^{*} \in B$ is a critical point of $J$ if $u^{*}$ solves its Euler-Lagrange equation $J^{\prime}(u)=0$, where $J^{\prime}(u)$ is its Fréchet derivative at $u$. $c=J\left(u^{*}\right)$ is called the critical level of $J$ at $u^{*}$. A critical point $u^{*}$ is nondegenerate if $J^{\prime \prime}\left(u^{*}\right)$ exists and has a bounded inverse. Otherwise $u^{*}$ is degenerate. The first candidates for critical points are the local extrema of $J$. Critical points that are not local extrema are called saddle points. In a physical system, saddle points appear as unstable equilibria or excited states.

Eigenpairs $(\lambda, u)$ of $(1.9)$ correspond to critical points of its Lagrange functional

$$
\mathcal{L}(u, \lambda)=F(u)-\lambda(G(u)-\alpha) .
$$

People used to be interested only in the stable solutions (ground states) to a physical system, which correspond to the local minima of its energy functional. Thus traditional existence analysis and numerical methods were focused on such solutions. Other solutions are unstable equilibria or excited states and correspond to saddle critical points of its energy functional. They are much harder for theoretical analysis and more elusive for numerical approximation. However, multiply/highly excited states have been observed in various physical systems to have a variety of configurations, instability/maneuverability. With new advanced laser (synchrotronic) technologies, scientists are able to induce and reach those excited unstable solutions and search for new applications [1, 25, 29, 28, 31]. Such advanced study and applications have changed people's view about unstable (excited) solutions. Thus a huge literature [12, 13 is available in modern critical point theory on the existence of multiple unstable solutions to various nonlinear equations including (1.3)-(1.6) and (1.10). For related numerical computations, a large literature exists in computational physics/chemistry. However most of these results are just for lowly (simply/doubly) excited states (see, e.g., a survey paper [11]). The methods are usually based upon physical intuition of a problem and are not mathematically justified. Although this type of problem did not gain enough attention in the computational mathematics community, we have witnessed the momentum building up in recent years; see $[2,5,26]$, and a series of publications, e.g., [8, 9], by Garcia-Ripoll and Perez-Garcia and their associates. Even numerical results for computing highly excited states have appeared more often recently $[1,6,17,26,34]$. However, most of them are based on a Newton-type method. They depend heavily on a good initial guess; i.e., an initial guess must be chosen close to the unknown solution to be found, they cannot handle degenerate cases and do not assume or use the variational structure of a problem, and thus are blind to instability order of multiple solutions. However, instability of excited states is an important issue in applications. So far, the only mathematically justified numerical method for computing multiple saddle points of a functional following their instability order is the local minimax (LMM) developed in [20, 21, 40, 41, 32, 33, 35, 36]. But it does not quite fit to NEP (1.10) due to the constraint.

For NEP (1.10), the simplest case is where both $F^{\prime}$ and $G^{\prime}$ are linear, e.g., $F^{\prime}(u)=$ $A u$ and $G^{\prime}(u)=u$, where $A$ is a linear self-adjoint operator from a Hilbert space $B$ to $B$. Then (1.10) is a well-studied linear eigenpair problem whose wide applications are well known with various numerical packages available. When either $F^{\prime}$ or $G^{\prime}$ is nonlinear, (1.10) is a nonlinear eigenpair problem. For example, in (1.4), $F^{\prime}$ is linear but $G^{\prime}$ is nonlinear; in (1.3), $G^{\prime}$ is linear but $F^{\prime}$ is nonlinear; and in (1.6), both $F^{\prime}$ and $G^{\prime}$ are nonlinear.

So far, people's understanding of (1.10) is still quite limited, and its exact solutions are too hard to obtain. Due to the space setting, the nonlinearity, and the 
multiplicity of solutions, (1.10) is very difficult to solve numerically. The first problem one has to overcome is how to numerically search for a new solution, not those known solutions. A saddle-type unstable solution may be easily perturbed to decay to a solution in a lower critical level. Whether or not a proposed numerical algorithm can find eigenfunctions following their instability order becomes an important issue. First, with an order, one can avoid a search going to those known solutions, and one can discuss whether or not some eigenfunctions have been missing in a computation. Second, since all eigenfunctions are unstable solutions, except those corresponding to local minima of the energy functional, their instability index is important information in an application. Since all eigenvalues in (1.10) are real, a natural order of eigenfunctions is the order of their eigenvalues. Then two questions must be answered: (a) Can a numerical algorithm find eigenfunctions in this order? If the answer to (a) is yes, then (b) is there a relation between this natural order and the instability order of their eigenfunctions? When (1.10) is linear and in a Hilbert space (Example 1.1)), due to the orthogonality between two different eigenfunctions, the Rayleigh-Ritz method (see [39] and Theorem 2.2) uses the orthogonal complement space of the known solutions to find eigenfunctions following the order of their eigenvalues. The eigenvalues are also the critical values of the Rayleigh quotient at their eigenfunctions. Thus this order coincides with the order of critical (energy) level of the eigenfunctions. Can one do it the same way when (1.10) is nonlinear in a Banach space, and the orthogonality between two different eigenfunctions is broken? Such a numerical method is not available so far in the literature.

LMM can be modified to find critical points of (1.11) following the order of their local minimax index (LMI) (an instability index) [41]. What is the relation between LMI of critical points of (1.11) and the order of eigenvalues of (1.10)? The literature does not provide any information. It turns out that in general this is a very tough question. Can we classify eigenpair problems that may provide us with such information? In a sequence of papers we will try to answer these questions. In this paper, we assume that the eigenpair problem (1.10) satisfies the iso-homogeneous condition; i.e., there are $k=l>0$ s.t.

$$
F^{\prime}(t u)=t^{k} F^{\prime}(u) \quad \text { and } \quad G^{\prime}(t u)=t^{l} G^{\prime}(u) \quad \forall t>0, u \in B .
$$

Example 1.1. A linear eigenpair problem. Find $(\lambda, u) \in \mathbb{R} \times(B \backslash\{0\})$ s.t. $F u=\lambda G u$, where $F$ and $G$ are self-adjoint linear operators on a Hilbert space $B$ and $G$ is also positive definite, bounded. Then the iso-homogeneous condition (1.12) is satisfied with $k=l=1$.

Equation (1.9) satisfies the iso-homogeneous condition (1.12) with $k=l=p-1$. Let $U=\{u \in B \mid G(u)=0\}$. For most iso-homogeneous NEP including (1.9), $U=\{0\}$; thus, to simplify our discussion, we assume $U=\{0\}$. Define the Rayleigh quotient

$$
J(u)=\frac{F(u)}{G(u)} \quad \forall u \in B \backslash U .
$$

Then $J \in C^{1}(B \backslash U, \mathbb{R})$. Even without the iso-homogeneous condition, critical points and their critical values of $J$ are always eigenfunctions and their eigenvalues of (1.10). The converse is not true in general. With the iso-homogeneous condition, it is proved in section 2 that the converse is also true. Thus we can focus on finding critical points of $J$. However, this problem has its own characters: due to the iso-homogeneous condition, $\alpha$ in (1.10) can be replaced by any nonzero number; i.e., if $u^{*}$ is an eigenfunction, then so is $t u^{*}$ for any $t>0$; i.e., no eigenfunction is isolated, and also 
$J(t u) \equiv J(u) \forall t>0$ and $u \in B \backslash U$. Thus $\left\langle J^{\prime}(u), u\right\rangle=0$ and $J^{\prime \prime}(u)$ is not invertible everywhere, a severe degenerate case. We limit $J$ on the unit sphere, and then the problem becomes constrained (still degenerate). We need to modify the LMM to solve for multiple critical points of $J$ restricted to a unit sphere following the order of their LMI. To do so, section 2 is used to develop a corresponding LMM characterization of critical points. Its relations with other characterizations such as Rayleigh-Ritz, Courant-Fischer, and Ljusternik-Schnirelman are also presented there. Based upon this LMM characterization, a modified LMM is devised in section 3. Then in section 4 , we implement the algorithm to solve our model problem (1.9). Except for the first eigenpair, which has been numerically computed in the literature [19], this is the first time that all of these eigenpairs have been numerically captured. Thus observations of those eigenfunctions on certain interesting phenomena are reported. In section 5, we present mathematical analysis related to convergence of the algorithm, an instability order of eigenfunctions, etc. In a subsequent paper [38], we solve (1.10) for nonhomogeneous cases, e.g., (1.3).

\section{Characterization of eigenpairs.}

\subsection{Equivalence between critical points and eigenpairs.}

LEMma 2.1. Under the iso-homogeneous condition, a pair $(\lambda, u)$ is an eigenpair of (1.10) if and only if $u$ is a critical point of $J$ and $\lambda=J(u)$ is the corresponding critical value.

Proof. The "if" part is always true. To see the "only if" part, let $u \in B \backslash U$. Then we have

$$
\int_{0}^{1}\left\langle F^{\prime}(t u), u\right\rangle d t=\int_{0}^{1} \frac{d}{d t} F(t u) d t=F(u)
$$

Similarly, $\int_{0}^{1}\left\langle G^{\prime}(t u), u\right\rangle d t=G(u)$. Thus, if $(\lambda, u)$ is an eigenpair of $(1.10)$, i.e., $F^{\prime}(u)=$ $\lambda G^{\prime}(u)$, then the iso-homogeneous condition implies $F^{\prime}(t u)=\lambda G^{\prime}(t u) \forall t>0$. This leads to $F(u)=\int_{0}^{1}\left\langle F^{\prime}(t u), u\right\rangle d t=\int_{0}^{1} \lambda\left\langle G^{\prime}(t u), u\right\rangle d t=\lambda G(u)$. Thus

$$
\lambda=\frac{F^{\prime}(u)}{G^{\prime}(u)}=\frac{F(u)}{G(u)} \equiv J(u) \quad \text { and } \quad J^{\prime}(u)=\frac{F^{\prime}(u) G(u)-F(u) G^{\prime}(u)}{G^{2}(u)}=0 .
$$

Remark 2.1. We can focus on finding critical points of $J$. An important consequence of Lemma 2.1 is that if critical points are found in such a way that their critical values are in a monotone (increasing) order, then eigenvalues are obtained in the same order. Thus it is easy for us to discuss whether or not we miss any eigenfunctions.

2.2. A modified local minimax characterization. To modify the original LMM, let us introduce the following related notions. Define $L=\left[u_{1}, u_{2}, \ldots, u_{n-1}\right]$ as the space spanned by linearly independent normalized $u_{1}, u_{2}, \ldots, u_{n-1} \in B$ and $B=L \oplus L^{\prime}$, where $L^{\prime}$ is the complement of $L$. Let $\mathcal{P}: B \rightarrow L^{\prime}$ be the corresponding linear projection operator and $S_{L^{\prime}}$ be the unit sphere in $L^{\prime}$. For each $u \in S_{L^{\prime}}$ denote $[L, u]_{S}=\left\{w=\sum_{k=1}^{n-1} t_{k} u_{k}+t_{0} u \mid \sum_{k=1}^{n-1} t_{k}^{2}+t_{0}^{2}=1\right\}$.

Definition 2.2. A set-valued mapping $P: S_{L^{\prime}} \rightarrow 2^{B}$ is the peak mapping of $J$ w.r.t. $L$ if, for each $u \in S_{L^{\prime}}, P(u)$ is the set of all local maximum points of $J$ on $[L, u]_{S}$; i.e., $w \in[L, u]_{S}$ is in $P(u)$ if and only if there is a neighborhood $\mathcal{N}(w)$ of $w$ s.t. $J(v) \leq J(w) \forall v \in[L, u]_{S} \cap \mathcal{N}(w)$. A single-valued mapping $p: S_{L^{\prime}} \rightarrow B$ is a peak selection of $J$ w.r.t. $L$ if $p(u) \in P(u) \forall u \in S_{L^{\prime}}$. For a given $u \in S_{L^{\prime}}, p$ is said to be $a$ local peak selection of $J$ at $u$ if the peak selection $p$ is locally defined near $u$. 
Since $J$ is a function continuous on the nonempty compact set $[L, u]_{S}$ for each $u \in S_{L^{\prime}}, P(u)$ is always nonempty. Such a defined peak selection has some interesting properties that will be frequently used.

Lemma 2.3. For each $u_{0} \in S_{L^{\prime}}$, if $p$ is a local peak selection of $J$ at $u_{0}$, then $\left\langle J^{\prime}\left(p\left(u_{0}\right)\right), u_{i}\right\rangle=0, i=0,1, \ldots, n-1$.

Proof. By the assumption, let $w=p\left(u_{0}\right)=\sum_{i=0}^{n-1} t_{i} u_{i} \in\left[L, u_{0}\right]_{S}$, where $\sum_{i=0}^{n-1} t_{i}^{2}=$ 1. For $0 \leq i \leq n-1$, if $\left\langle J^{\prime}(w), u_{i}\right\rangle \neq 0$, let $w(s)=\frac{w+s u_{i}}{c(s)} \in\left[L, u_{0}\right]_{S}$, where $c(s)=\left[\left(t_{i}+s\right)^{2}+\sum_{k=0, k \neq i}^{n-1} t_{k}^{2}\right]^{\frac{1}{2}}$. Since $w(s) \rightarrow w$ as $s \rightarrow 0$, there exists $s_{0}>0$ s.t. when $0<|s|<s_{0}$, we have

$$
J(w(s))-J(w)=\left\langle J^{\prime}(w), \frac{s}{c(s)} u_{i}\right\rangle+o(\|w(s)-w\|),
$$

where we have used the fact that $\left\langle J^{\prime}(w), w\right\rangle=0$. Thus when $|s|$ is small, the term $\frac{s}{c(s)}\left\langle J^{\prime}(w), u_{i}\right\rangle$ dominates the difference of $J(w(s))-J(w)$. Since this term can be made either positive or negative as we wish by properly selecting $s \neq 0$, it contradicts the assumption that $w$ is a local maximum of $J$ on $\left[L, u_{0}\right]_{S}$. Thus $\left\langle J^{\prime}(w), u_{i}\right\rangle=0$.

Since $J^{\prime}(u) \in B^{\prime}$ not $B$, to find a descent direction, the role of the gradient in a Hilbert space is replaced by that of a pseudogradient in a Banach space.

Definition 2.4. Let $\bar{B}=\left\{u \in B: J^{\prime}(u) \neq 0\right\}$ and $\theta \in(0,1]$. For $u \in \bar{B}$, a vector $\mathcal{G}(u) \in B$ is a pseudogradient of $J$ at $u$ w.r.t. $\theta$ if

$$
\|\mathcal{G}(u)\| \leq 1 \quad \text { and } \quad\left\langle J^{\prime}(u), \mathcal{G}(u)\right\rangle \geq \theta\left\|J^{\prime}(u)\right\|
$$

If such $\mathcal{G}$ is continuous, then $\mathcal{G}$ is called a pseudogradient flow.

The following lemma leads to a new LMM characterization of critical points of $J$ and also a stepsize rule in our modified LMM method; see Step 4 of the flow chart in section 3 .

Lemma 2.5. Let a local peak selection $p$ of $J$ be continuous at $\bar{u} \in S_{L^{\prime}}$ with $J^{\prime}(p(\bar{u})) \neq 0$ and $p(\bar{u}) \notin L$. When $s>0$ is small and $\bar{u}(s)=\frac{\bar{u}+s w(\bar{u})}{\|\bar{u}+s w(\bar{u})\|} \in S_{L^{\prime}}$, we have

$$
J(p(\bar{u}(s)))<J(p(\bar{u}))-\frac{1}{4} s \theta\left|t_{n}\right|\left\|J^{\prime}(p(\bar{u}))\right\| \text {, where } p(\bar{u})=\sum_{i=1}^{n-1} t_{i} u_{i}+t_{n} \bar{u} \in[L, \bar{u}]_{S},
$$

i.e., $\sum_{k=1}^{n} t_{k}^{2}=1, w(\bar{u})=-\operatorname{sign}\left(t_{n}\right) \mathcal{P}(\mathcal{G}(p(\bar{u})))$, and $\mathcal{P}(\mathcal{G}(p(\bar{u})))$ is the projection of a pseudogradient of $J$ at $p(\bar{u})$ with a constant $\theta \in(0,1]$.

Proof. Since $\left\langle J^{\prime}(w), w\right\rangle=0 \forall w \in B \backslash U, p$ is continuous at $\bar{u}$, and $p(\bar{u}) \in[L, \bar{u}]_{S}$ when $s>0$ is small, we have

$$
\begin{aligned}
J(p(\bar{u}(s))) & =J(p(\bar{u}))+\left\langle J^{\prime}(p(\bar{u})), p(\bar{u}(s))-p(\bar{u})\right\rangle+o(\|p(\bar{u}(s)-p(\bar{u}))\|) \\
& =J(p(\bar{u}))+\left\langle J^{\prime}(p(\bar{u})), p(\bar{u}(s))\right\rangle+o(\|p(\bar{u}(s)-p(\bar{u}))\|) .
\end{aligned}
$$

Since $p(\bar{u}) \notin L$ implies $t_{n}>0$ and, by Lemma 2.3 , as $s>0$ is small, we have $t_{n}(s)>0$ and

$$
\left\langle J^{\prime}(p(\bar{u})), p(\bar{u}(s))\right\rangle=-\frac{\operatorname{sign}\left(t_{n}\right) t_{n}(s) s}{\|\bar{u}+s w(\bar{u})\|}\left\langle J^{\prime}(p(\bar{u})), \mathcal{P}(\mathcal{G}(p(\bar{u})))\right\rangle<-\frac{1}{2} s \theta\left|t_{n}\right|\left\|J^{\prime}(p(\bar{u}))\right\|,
$$

where $p(\bar{u}(s)) \equiv p\left(\frac{\bar{u}+s w(\bar{u})}{\|\bar{u}+s w(\bar{u})\|}\right)=t_{1}(s) u_{1}+\cdots+t_{n-1}(s) u_{n-1}+t_{n}(s) \frac{\bar{u}+s w(\bar{u})}{\|\bar{u}+s w(\bar{u})\|} \in$ $[L, \bar{u}(s)]_{S}$. Hence, when $s>0$ is small,

$$
J(p(\bar{u}(s)))<J(p(\bar{u}))-\frac{1}{4} s \theta\left|t_{n}\right|\left\|J^{\prime}(p(\bar{u}))\right\| .
$$


Remark 2.2. From the last two lemmas, it is clear that the notion of a peak selection $p(\bar{u})$ can be generalized to satisfy $\left\langle J^{\prime}(p(\bar{u})), \bar{u}\right\rangle=\left\langle J^{\prime}(p(\bar{u})), u_{i}\right\rangle=0, i=$ $1, \ldots, n-1$. As a direct consequence of Lemma 2.5, we have the following LMM characterization.

THEOREM 2.6. Assume that a local peak selection $p$ of $J$ is continuous at $\bar{u} \in S_{L^{\prime}}$. If $\bar{u}=\arg$ local- $\min _{u \in S_{L^{\prime}}} J(p(u))$ and $\operatorname{dis}(p(\bar{u}), L)>0$, then $p(\bar{u})$ is a critical point of $J$; i.e., $p(\bar{u})$ is an eigenfunction of (1.10) and $\lambda=J(p(\bar{u}))$ is the corresponding eigenvalue.

2.3. Comparison to other characterizations. Denote the generalized solution set $\mathcal{M}=\left\{p(u) \mid u \in S_{L^{\prime}}\right\}$. Theorem 2.6 states that the $n$th eigenfunction $u_{n}$ can be obtained through finding a local minimum of $J$ on $\mathcal{M}$ or solving

$$
\min _{u \in S_{L^{\prime}}} \max _{v \in\left[u_{1}, \ldots, u_{n-1}, u\right]_{S}} J(v) .
$$

Next we discuss the relation between (2.1) and other well-known minimax principles.

THEOREM 2.7. For the linear eigenpair problem, Example 1.1 in a Hilbert space $B$, the modified LMM (2.1) is equivalent to the Rayleigh-Ritz method [39]; i.e., by letting $\langle u, v\rangle_{G}=\langle G u, v\rangle$ and $\|u\|_{G}=\left(\langle u, u\rangle_{G}\right)^{\frac{1}{2}}$ be the equivalent inner product and norm on $B, L^{\prime}=L^{\perp}=\left\{u \in B \mid\left\langle u, u_{i}\right\rangle_{G}=0, i=1, \ldots, n-1\right\}$ and $S_{L^{\perp}}=\left\{u \in L^{\perp}\right.$ : $\left.\|u\|_{G}=1\right\}$, then

$$
u_{n}=\arg \min _{u \in S_{L \perp}} \max _{v \in\left[u_{1}, \ldots, u_{n-1}, u\right]_{S}} J(v)=\arg \min _{u \in S_{L^{\perp}}} J(u) .
$$

Proof. It is known that $\left\langle u_{i}, u_{j}\right\rangle_{G}=0,1 \leq i<j \leq n$. Thus we only have to find $v=p(u) \in L^{\perp}$. Then (2.1) reduces to the Rayleigh-Ritz orthogonal method

$$
\min _{u \in S_{L^{\perp}}} \max _{v \in[u]_{S}} J(v)=\min _{u \in S_{L^{\perp}}} J(u) .
$$

Conversely, if $u_{n}=\arg \min _{u \in S_{L \perp}} J(u)=\arg \min _{u \in S_{L^{\perp}}}\langle F u, u\rangle$, i.e., $u_{n}$ is found by the Rayleigh-Ritz method, then there is a neighborhood $\mathcal{N}\left(u_{n}\right)$ of $u_{n}$ s.t. $\forall u \in \mathcal{N}\left(u_{n}\right) \cap$ $S_{L^{\perp}}$

$$
\max _{v \in\left[u_{1}, \ldots, u_{n-1}, u\right]_{S}}\langle F v, v\rangle \geq\langle F u, u\rangle \geq\left\langle F u_{n}, u_{n}\right\rangle .
$$

In addition, $\forall u \in\left[u_{1}, \ldots, u_{n-1}, u_{n}\right]_{S}$, we have $u=\sum_{i=1}^{n} c_{i} u_{i}$ with $\sum_{i=1}^{n} c_{i}^{2}=1$. Then

$$
\begin{aligned}
& J(u)=\langle F u, u\rangle \\
= & \sum_{i=1}^{n} \sum_{j=1}^{n} c_{i} c_{j}\left\langle F u_{i}, u_{j}\right\rangle=\sum_{i=1}^{n} \sum_{j=1}^{n} c_{i} c_{j} \lambda_{i}\left\langle G u_{i}, u_{j}\right\rangle=\sum_{i=1}^{n} c_{i}^{2} \lambda_{i} \leq \lambda_{n}=J\left(u_{n}\right),
\end{aligned}
$$

where $\lambda_{i}$ is the eigenvalue corresponding to $u_{i}$. Therefore

$$
\begin{aligned}
J\left(u_{n}\right) & =\left\langle F u_{n}, u_{n}\right\rangle=\min _{u \in S_{L \perp}} \max _{v \in\left[u_{1}, \ldots, u_{n-1}, u_{n}\right]_{S}}\langle F v, v\rangle \\
& =\min _{u \in S_{L \perp}} \max _{v \in\left[u_{1}, \ldots, u_{n-1}, u_{n}\right]_{S}} J(v) .
\end{aligned}
$$

As for the NEP, since the orthogonality between two different eigenfunctions is broken, the Courant-Fischer minimax principle [39] states that $u_{n}$ can be found by solving

$$
\min _{W_{n}} \max _{v \in W_{n} \cap S} J(v),
$$


where min is taken over all the subspaces $W_{n}$ of dimension $n$ in $B$, and $\max$ is global on $W_{n} \cap S$. The Courant-Fischer minimax principle is originally designed for linear eigenpair problems. People then found that it was also valid for nonlinear eigenpair problems where the iso-homogeneous condition is satisfied. The LjusternikSchnirelman minimax principle [39], which is commonly regarded as a generalization of the Courant-Fischer minimax principle, is used to characterize saddle points of a nonlinear functional $J$ through solving

$$
\inf _{K \in \mathcal{K}_{n}} \sup _{u \in K} J(u)
$$

where $\mathcal{K}_{n}$ is the class of all compact subsets $K$ of $B$ with $\operatorname{ind}(K) \geq n$, sup is global on $K$, and inf is global over $\mathcal{K}_{n}$. When the iso-homogeneous condition is satisfied and $J$ is the Rayleigh quotient, (2.4) coincides with (2.3). However, they are all two-level global minimax characterizations and do not help much in algorithm implementation. In contrast, our LMM method (2.1) can be implemented as the following numerical algorithm.

3. A modified LMM algorithm. Let $u_{1}, \ldots, u_{n-1}$ be $n-1$ previously found normalized critical points of $J, L=\left[u_{1}, \ldots, u_{n-1}\right], B=L \oplus L^{\prime}$, and $\mathcal{P}: B \rightarrow L^{\prime}$ be the corresponding linear continuous projection operator.

Step 1. Given $\theta \in(0,1)$ and $\lambda>0$. Set $k=1$. Choose $v_{n}^{1} \in S_{L^{\prime}}$, and solve for

$$
u_{n}^{1} \equiv p\left(v_{n}^{1}\right) \equiv \sum_{i=1}^{n-1} t_{i}^{1} u_{i}+t_{n}^{1} v_{n}^{1} \equiv \arg \max \left\{J(v) \mid v=\sum_{i=1}^{n-1} t_{i} u_{i}+t_{n} v_{n}^{1}, \sum_{i=1}^{n} t_{i}^{2}=1\right\} .
$$

Step 2. Compute a descent direction $w_{n}^{k}=-\operatorname{sign}\left(t_{n}^{k}\right) \mathcal{P}\left(\mathcal{G}_{n}^{k}\right)$, where $\mathcal{G}_{n}^{k}$ is a pseudogradient of $J$ at $u_{n}^{k}$ with constant $\theta$.

Step 3. If $\left\|J^{\prime}\left(p\left(v_{n}^{k}\right)\right)\right\| \leq \epsilon$, then output $u_{n}^{k}=p\left(v_{n}^{k}\right)$ and stop. Otherwise, do Step 4.

Step 4. Define $v_{n}^{k}(s)=\frac{v_{n}^{k}+s w_{n}^{k}}{\left\|v_{n}^{k}+s w_{n}^{k}\right\|}$ and

$$
u_{n}^{k}(s) \equiv p\left(v_{n}^{k}(s)\right) \equiv \arg \max \left\{J(v) \mid v=\sum_{i=1}^{n-1} t_{i} u_{i}+t_{n} v_{n}^{k}(s), \sum_{i=1}^{n} t_{i}^{2}=1\right\},
$$

where $\left(t_{1}^{k}, \ldots, t_{n}^{k}\right)$ is used as an initial point. Determine the stepsize

$$
s_{n}^{k}=\max _{m \in N}\left\{\frac{\lambda}{2^{m}} \mid 2^{m} \geq\left\|w_{n}^{k}\right\|, J\left(p\left(v_{n}^{k}\left(\frac{\lambda}{2^{m}}\right)\right)\right)-J\left(u_{n}^{k}\right) \leq-\frac{s \theta\left|t_{n}^{k}\right|}{4}\left\|J^{\prime}\left(u_{n}^{k}\right)\right\|\right\} .
$$

Step 5. Set $v_{n}^{k+1}=v_{n}^{k}\left(s_{n}^{k}\right), u_{n}^{k+1}=u_{n}^{k}\left(s_{n}^{k}\right) \equiv p\left(v_{n}^{k+1}\right) \equiv \sum_{i=1}^{n-1} t_{i}^{k+1} u_{i}+t_{n}^{k+1} v_{n}^{k}\left(s_{n}^{k}\right)$. $k=k+1$ and go to Step 2 .

Remark 3.1. (a) In Step 1, since $L$ is finite-dimensional, we may either use a decomposition and normalization to choose $v_{n}^{1} \in S_{L^{\prime}}$ or use observation, among all functions "orthogonal" to $L$, to choose a normalized $v_{n}^{1}$ with the simplest nodal line structure. Our choice does not have to be exact. For example, when $L=\{0\}, v_{1}^{1}$ can be any nonzero function, but a positive one has the simplest nodal line structure. When $L=\left[u_{1}\right]$, where $u_{1}$ is positive, among all functions orthogonal to $L$ we choose $v_{2}^{1}$ to be sign-changing and normalized with one nodal line, etc.

(b) In Step 2, theoretically we can either follow a pseudogradient flow or just find a pseudogradient. The projection is important to avoid a decay to the trivial/known 
solutions, i.e., to satisfy $u^{*}=p\left(v^{*}\right) \notin L$. For a practical technique to compute the projection of a pseudogradient to a complement subspace of $L$ in case of $B=W^{1, p}(\Omega)$, see section 4.1 .

(c) In Step 4, it is important to use the initial guess $\left(t_{1}^{k}, \ldots, t_{n}^{k}\right)$ in order to consistently trace a peak selection and keep it continuous. by

(d) It is easy to check that $\frac{1}{2} s^{k} \leq s_{n}^{k} \leq s^{k}$, where $s^{k}$ is the exact stepsize defined

$$
s^{k}=\max \left\{s\left|J\left(p\left(v_{n}^{k}(s)\right)\right)-J\left(u_{n}^{k}\right) \leq-\frac{1}{4} s \theta\right| t_{n}^{k} \mid\left\|J^{\prime}\left(u_{n}^{k}\right)\right\|, \lambda \geq s\left\|w_{n}^{k}\right\| \geq 0\right\}
$$

and is actually used in our convergence analysis [36].

(e) The algorithm is stable in the sense $J\left(u_{n}^{k+1}\right)<J\left(u_{n}^{k}\right)$.

4. Some numerical results for eigenpairs of the p-Laplacian. In this section we implement the modified LMM to numerically solve our model problem (1.9) for multiple eigenpairs. The Rayleigh quotient is of the form

$$
J(u)=\frac{\int_{\Omega}|\nabla u(x)|^{p} d x}{\int_{\Omega} w(x)|u(x)|^{p} d x}, \quad u \in B=W_{0}^{1, p}(\Omega), u \neq 0 .
$$

4.1. A practical technique for computing a pseudogradient. We develop a practical technique for computing the projection of a pseudogradient to a complement subspace of $L$ based on the gradient of $J$ and successfully apply it to all our numerical computations. The idea is described below.

The gradient $J^{\prime}(u)$ of $J$ at $u \in B$ is in the dual space $B^{\prime}=W^{-1, q}(\Omega),\left(\frac{1}{p}+\right.$ $\frac{1}{q}=1$ ), where the smoothness is poor, and thus not qualified as a candidate for a pseudogradient in $B$. To increase its smoothness, we use $\nabla J$ instead of $J^{\prime}$, where $d=\nabla J(u)$ is in $W_{0}^{1, q}(\Omega)$ and defined by $\langle d, v\rangle_{W_{0}^{1, q} \times W_{0}^{1, p}}=\left\langle J^{\prime}(u), v\right\rangle_{W^{-1, q} \times W_{0}^{1, p}} \forall v \in$ $W_{0}^{1, p}(\Omega)$, or

$$
\int_{\Omega} \nabla d(x) \cdot \nabla v(x) d x=-\int_{\Omega} \Delta d(x) v(x) d x=\int_{\Omega} J^{\prime}(u)(x) v(x) d x \quad \forall v \in W_{0}^{1, p}(\Omega),
$$

where $J^{\prime}(u)(x)=\frac{p}{b^{2}}\left(b \Delta_{p} u(x)+a w(x)|u(x)|^{p-2} u(x)\right)$ when $J$ is given by (4.1). This leads us to solve

$$
-\Delta d(x)=J^{\prime}(u)(x)=\frac{p}{b^{2}}\left(b \Delta_{p} u(x)+a w(x)|u(x)|^{p-2} u(x)\right), x \in \Omega, \quad \text { and }\left.\quad d\right|_{\partial \Omega}=0,
$$

where $a=\int_{\Omega}|\nabla u(x)|^{p} d x$ and $b=\int_{\Omega} w(x)|u(x)|^{p} d x$. Since in a numerical computation "nice" functions are usually used to approximate a solution, we check the ratio

$$
\gamma=\frac{\|d\|_{2}^{2}}{\|d\|_{p} \cdot\|d\|_{q}}
$$

where $\|\cdot\|_{r}$ is the norm in $W_{0}^{1, r}(\Omega)$. By the Holder inequality, $\gamma \leq 1$. If $\gamma>\alpha>0$ at all points generated by the algorithm, then $\mathcal{G}(u)=\frac{d}{\|d\|_{p}} \in W_{0}^{1, p}(\Omega) \cap W_{0}^{1, q}(\Omega)$ is a pseudogradient of $J$ at $u$ w.r.t. $\alpha$, since $\left\|J^{\prime}(u)\right\| \leq\|d\|_{q}$ [36]. All our numerical results show that $\gamma \gg 0$, although it has not been mathematically proved. As for the projection of $\mathcal{G}(p(u))=\frac{d}{\|d\|_{p}}$, where $d=\nabla J(p(u))$, to a complement subspace of $L$, by the definition of $p$, we note that

$$
0=\left\langle J^{\prime}(p(u)), v\right\rangle_{W^{-1, q} \times W_{0}^{1, p}}=\langle d, v\rangle_{W_{0}^{1, q} \times W_{0}^{1, p}} \quad \forall v \in L
$$


i.e., $d$ is "orthogonal" to $L$ in some sense. Thus no projection is needed. For such a pseudogradient, to get related convergence of the algorithm [36], in the flow chart, the expression of $w_{n}^{k}$ in Step 2 should be $w_{n}^{k}=-\operatorname{sign}\left(t_{n}^{k}\right) \nabla J\left(u_{n}^{k}\right)$, and the inequality to decide $s_{n}^{k}$ in Step 4 and (3.1) should be $J\left(p\left(v_{n}^{k}\left(\frac{\lambda}{2^{m}}\right)\right)\right)-J\left(u_{n}^{k}\right) \leq-\frac{\left|t_{n}^{k}\right|}{4}\left\|\nabla J\left(u_{n}^{k}\right)\right\|_{2}^{2}$. Then the expression of $w(\bar{u})$ in Lemma 2.5 should be $w(\bar{u})=-\operatorname{sign}\left(t_{n}\right) \nabla J(\bar{u})$, and under the assumption $\|\nabla J(p(\bar{u}))\|_{2}<+\infty$, the inequality should be $J(p(\bar{u}(s)))<$ $J(p(\bar{u}))-\frac{1}{4} s\left|t_{n}\right|\|\nabla J(p(\bar{u}))\|_{2}^{2}$.

4.2. Some numerical results and observations. We select $\Omega=[0,2] \times[0,2]$, $p=1.75,2.5,3.0$ for $w(x) \equiv 1$ and $p=1.75,2.5, r=0.5,6.0$ for $w(x)=|x-\overline{1}|^{r}$, where $\overline{1}=(1, \ldots, 1) \in \mathbb{R}^{n}$ and $|\cdot|$ is the Euclidean norm in $\mathbb{R}^{n}$. Either $800 \times 800$ or $1000 \times 1000$ linear square elements are used in the computation, and $\left\|\nabla J\left(p\left(v_{n}^{k}\right)\right)\right\| \leq \varepsilon=10^{-3}$ is used to terminate the iteration. In Figures 1-14, for each case, we display the profiles of the first few numerical eigenfunctions following the order of their LMI. If two eigenfunctions have the same LMI, we list the one with a smaller eigenvalue first.

In the remaining part of this section, let us shorten the notation: (right) by (r), (middle) by (m), and (left) by (l). In Figures 1(l), 3(m), 5(r), 8(l), 9(r), 11(m), and $13(\mathrm{l})$ are the ground states (LMI $=1)$ computed with $L=\{0\}$. For other solutions, due to higher LMI, for computational efficiency, we utilized their symmetries. For example, for Figures 3(l), 5(l), 7(m), 9(m), 11(l), 12(r), and 14(r), we used their symmetries with $L=\left[u_{1}\right]$, where $u_{1}$ is their ground state, and other solutions are computed by using their symmetries with $L=\{0\}$. See [32] for details. Several interesting phenomena related to the (weighted) eigenpairs of $\Delta_{p}$ on the square domains have been observed from our numerical results.

(a) By comparing Figure 8(l) to Figure 9(r) and Figure 11(m) to Figure 13(l), we see that a peak-breaking phenomenon takes place for the first weighted eigenfunctions of $\Delta_{p}$ as $r$ increases.

(b) By comparing eigenfunctions in each of the following groups: Figures 1(l), $3(\mathrm{~m})$, and 5(l); Figures $1(\mathrm{~m}), 4(\mathrm{l})$, and $6(\mathrm{~m})$; Figures $1(\mathrm{r}), 3(\mathrm{r})$, and $6(\mathrm{l})$; Figures 2(l), $4(\mathrm{~m})$, and $6(\mathrm{r})$; Figures $2(\mathrm{~m}), 5(\mathrm{~m})$, and $7(\mathrm{r})$; Figures 2(r), 4(r), and 7(l); Figures 3(l), $5(\mathrm{l})$, and $7(\mathrm{~m})$, we observe that for different values of $p$, they have the same number of peaks, similar peak locations, and peaks that become sharper when $p$ gets larger.

(c) Keeping the order of eigenvalues in mind and grouping them by their patterns, Figures 1(m), 4(l), and 6(m) (side-to-side peak); Figures 1(r), 3(r), and 6(l) (cornerto-corner peak); Figures $2(\mathrm{~m}), 5(\mathrm{~m})$, and 7(r) (3-peak); Figures 2(r), 4(r), and 7(l) (4-side-peak); Figures $3(\mathrm{l}), 5(\mathrm{l})$, and $7(\mathrm{~m})$ (5-peak), we find a very interesting pattern order switching phenomenon when $p$ crosses 2 , worth further investigation. Those phenomena also imply that when $p=2$, the eigenvalues of the eigenfunctions with the same LMI should be the same. To see this phenomenon more clearly, we plot the first three eigenvalues of $\Delta_{p}$ for $1.6 \leq p \leq 2.4$ in Figure 15 .

It is clear that the second and the third eigenfunctions of $\Delta_{p}$ switch their pattern order when $p$ crosses 2. That is why the second eigenvalue of $\Delta$ is double. To our surprise, from the plot, the first eigenvalue of $\Delta_{p}$ seems to have a linear relation in $p$. This is the first time such relations have been numerically captured. They are certainly interesting and worthy of further theoretical analysis.

(d) If we pay attention to the peak locations and compare Figure 8(l) to Figure 9(r), Figure 8(m) to Figure 10(l), Figure 9(m) to Figure 11(l), Figure 11(m) to Figure 13(l), Figure 12(l) to Figure 13(r), and Figure 12(r) to Figure 14(r), we can see that the peaks prefer the corners when $r$ increases and crosses some numbers. 

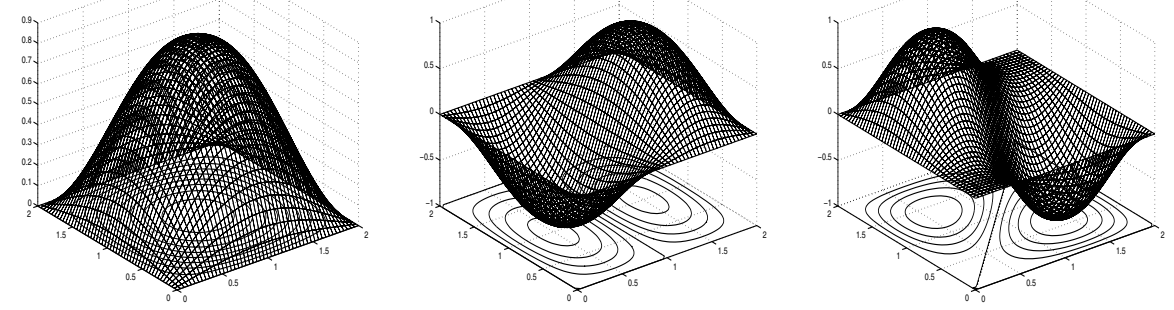

FIG. 1. Eigenfunctions of $\Delta_{p}, p=1.75: \lambda_{1}=4.245837$ (left), $\lambda_{2}=9.317313$ (middle), and $\lambda_{3}=9.407816$ (right).
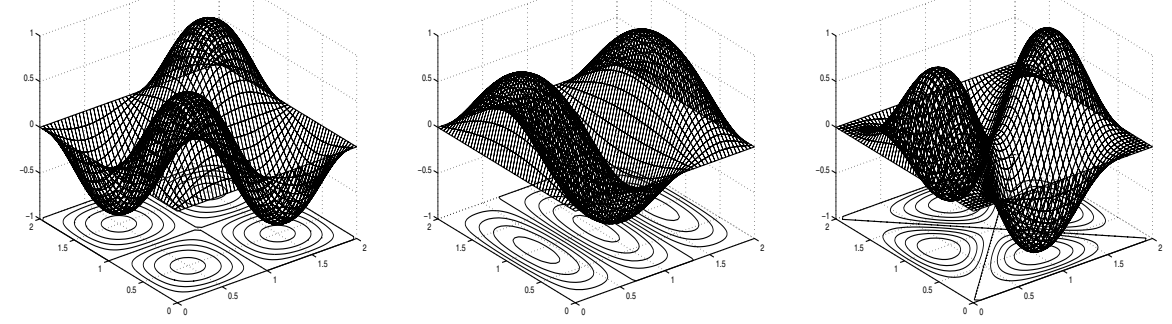

FIG. 2. Eigenfunctions of $\Delta_{p}, p=1.75: \lambda_{4}=14.280496$ (left), $\lambda_{5}=16.837822$ (middle), and $\lambda_{6}=17.254568$ (right).
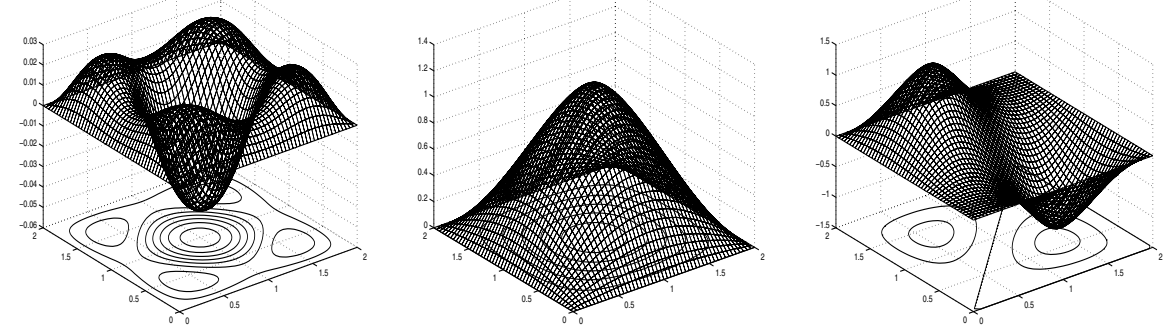

FIG. 3. Eigenfunctions of $\Delta_{p}, p=1.75: \quad \lambda_{7}=23.366003$ (left); $p=2.5, \lambda_{1}=6.354715$ (middle); and $\lambda_{2}=20.289627$ (right).
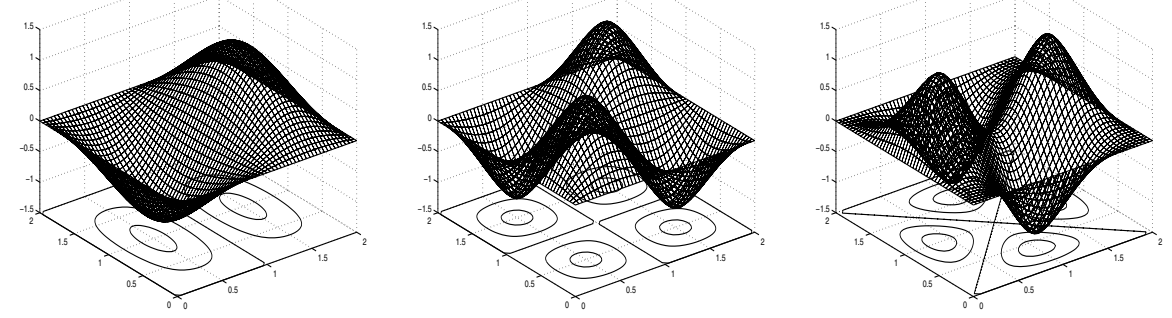

FIG. 4. Eigenfunctions of $\Delta_{p}, p=2.5: \lambda_{3}=20.798476$ (right), $\lambda_{4}=35.944786$ (middle), and $\lambda_{5}=48.259806$ (right). 

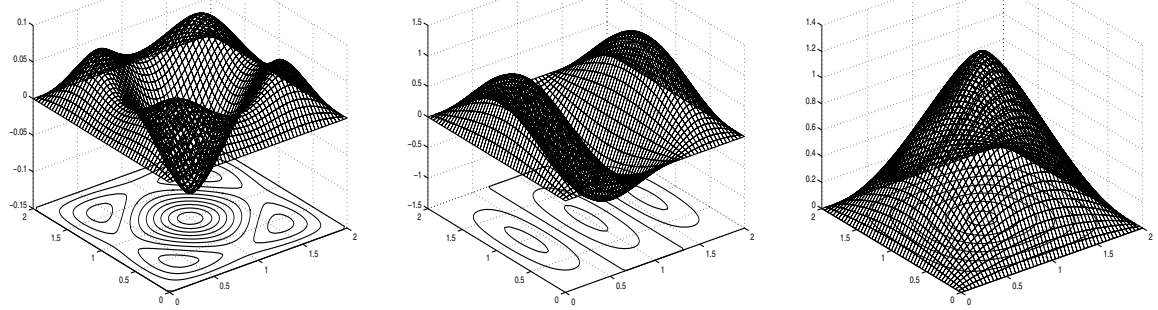

FIG. 5. Eigenfunctions of $\Delta_{p}, p=2.5: \lambda_{6}=49.679394$ (left), and $\lambda_{7}=51.104811$ (middle); $p=3.0, \lambda_{1}=7.844420$ (right).
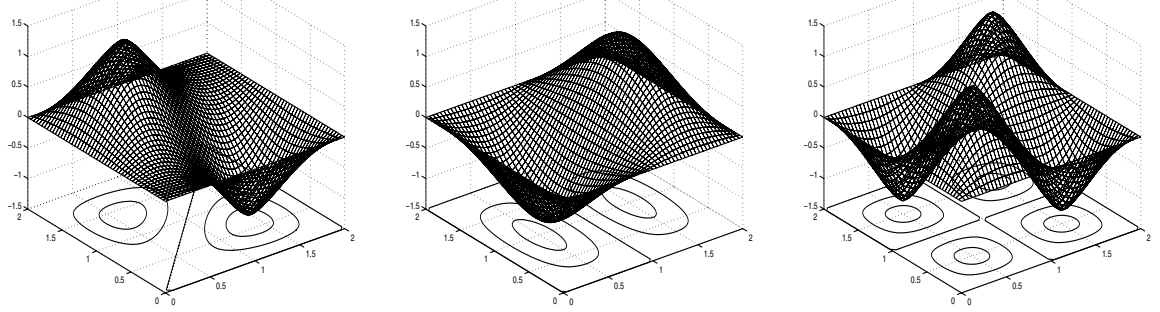

FIG. 6. Eigenfunctions of $\Delta_{p}, p=3.0: \lambda_{2}=32.098661$ (left), $\lambda_{3}=33.947805$ (middle), and $\lambda_{4}=62.748593$ (right).
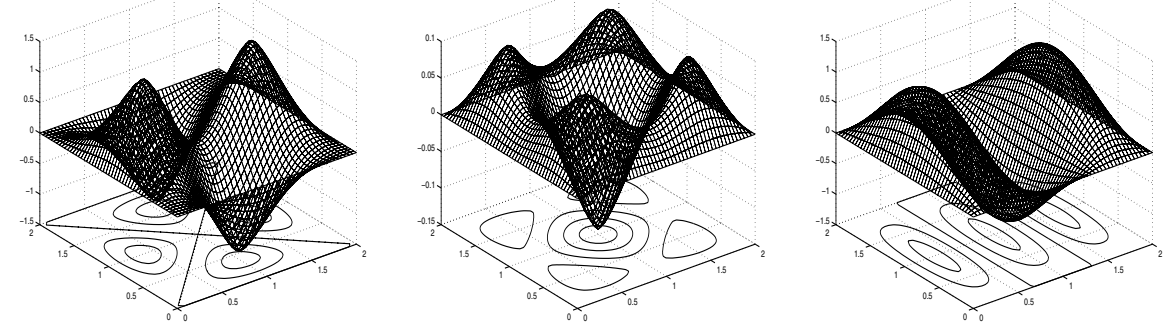

FIG. 7. Eigenfunctions of $\Delta_{p}, p=3.0: \lambda_{5}=90.795294$ (left), $\lambda_{6}=94.932100$ (middle), and $\lambda_{7}=102.660394$ (right).
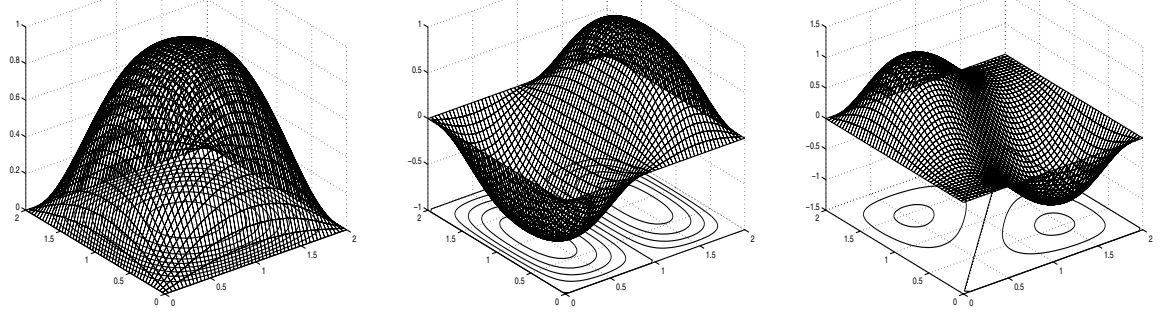

FIG. 8. Weighted eigenfunctions of $\Delta_{p}, p=1.75, r=0.5: \lambda_{1}=6.088006$ (left), $\lambda_{2}=11.775095$ (middle), and $\lambda_{3}=11.938270$ (right). 

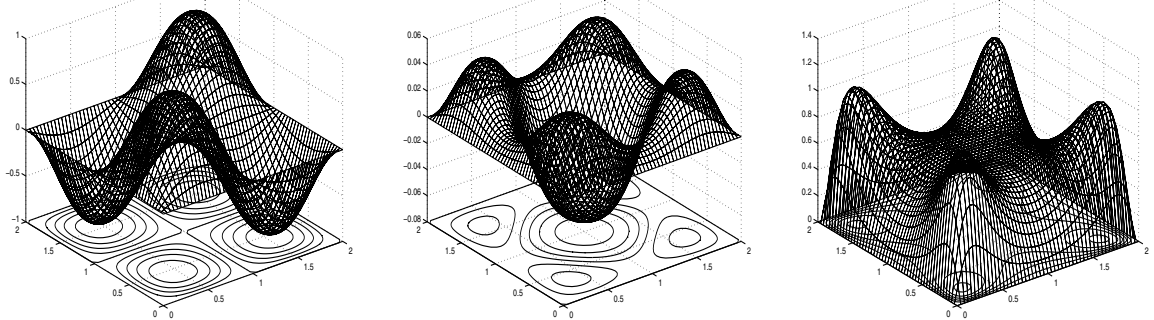

FIG. 9. Weighted eigenfunctions of $\Delta_{p}, p=1.75, r=0.5: \lambda_{4}=16.633820$ (left), and $\lambda_{5}=$ 23.366003 (middle); and $p=1.75, r=6.0, \lambda_{1}=18.714875$ (right).
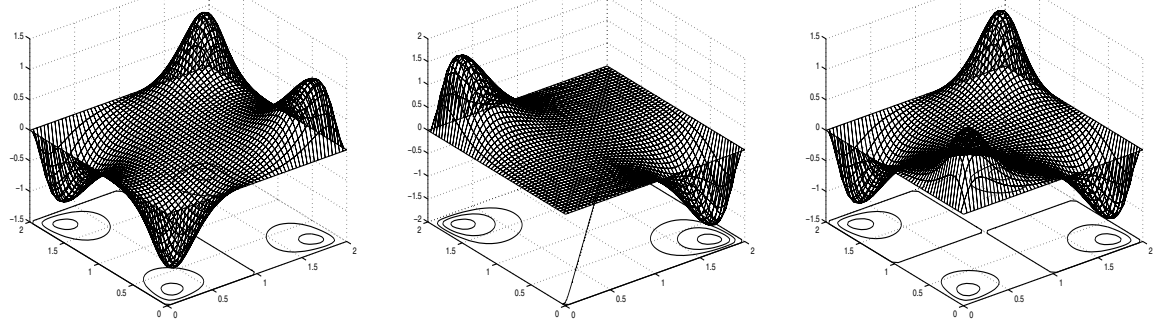

FIG. 10. Weighted eigenfunctions of $\Delta_{p}, p=1.75, r=6.0: \lambda_{2}=20.312840$ (left), $\lambda_{3}=$ 20.425545 (middle), and $\lambda_{4}=20.738396$ (right).
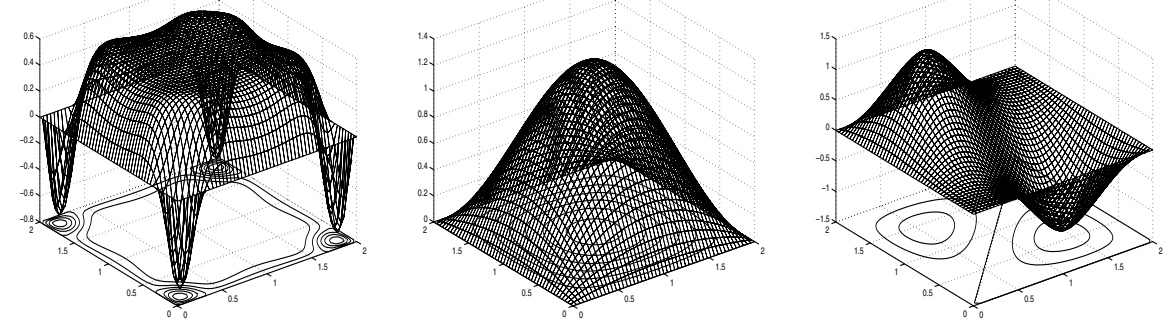

FIG. 11. Weighted eigenfunctions of $\Delta_{p}: p=1.75, r=6.0, \lambda_{5}=34.801623$ (left); $p=2.5$, $r=0.5, \lambda_{1}=10.185286$ (middle), and $\lambda_{2}=26.174362$ (right).
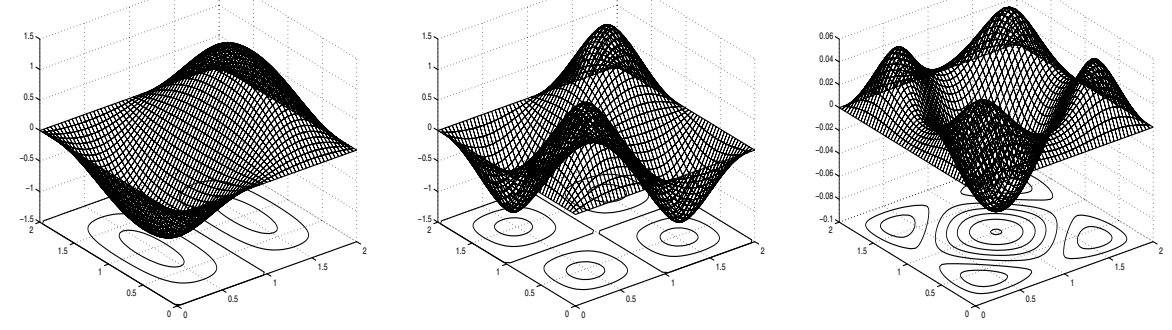

FIG. 12. Weighted eigenfunctions of $\Delta_{p}, p=2.5, r=0.5: \lambda_{3}=26.991732$ (left), $\lambda_{4}=$ 42.140740 (middle), and $\lambda_{5}=69.931326$ (right). 

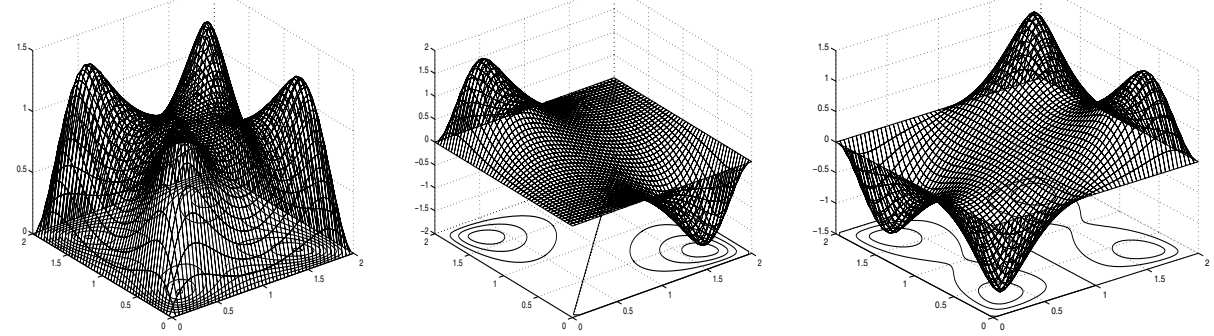

FIG. 13. Weighted eigenfunctions of $\Delta_{p}, p=2.5, r=6.0: \lambda_{1}=65.223275$ (left), $\lambda_{2}=$ 70.878805 (middle), and $\lambda_{3}=71.815461$ (right).
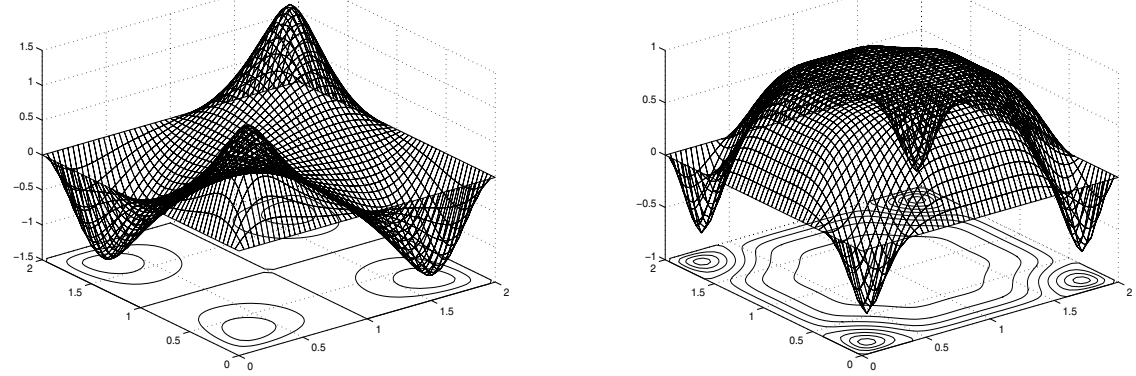

FIG. 14. Weighted eigenfunctions of $\Delta_{p}, p=2.5, r=6.0: \lambda_{4}=74.271235$ (left) and $\lambda_{5}=$ 161.729721 (right).

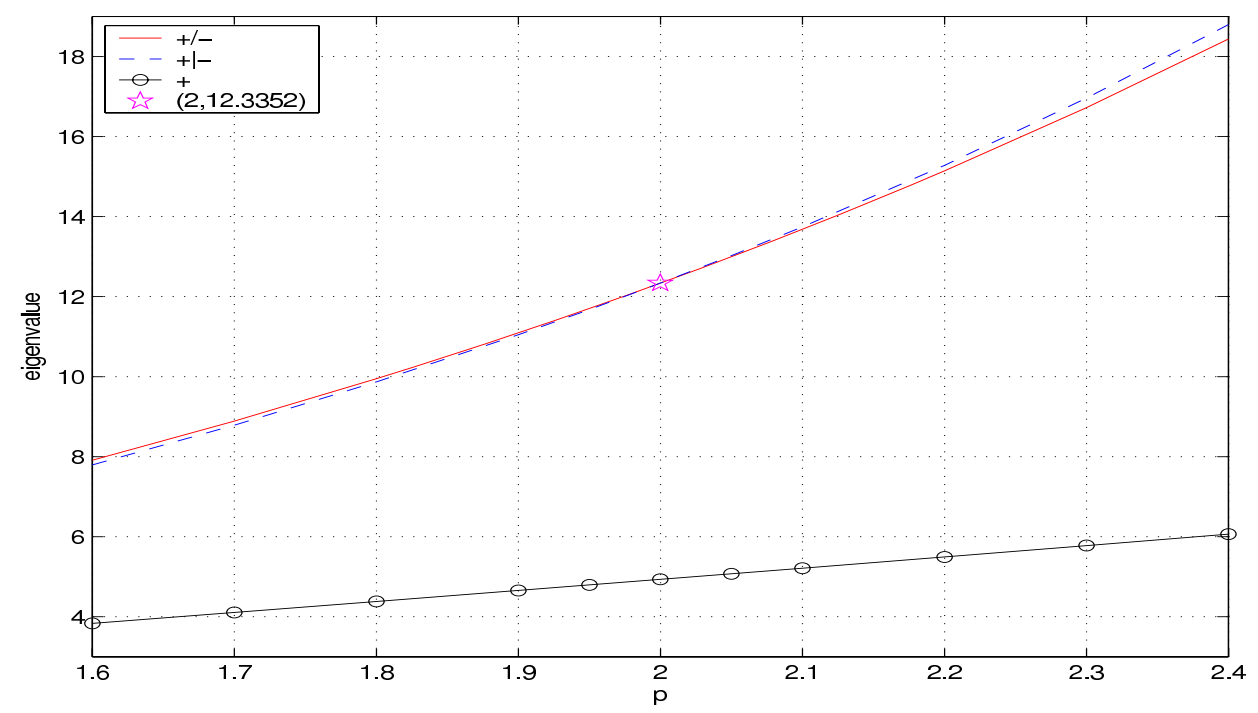

FIG. 15. The first three eigenvalues of $\Delta_{p}$ for $1.6 \leq p \leq 2.4$.

(e) To the weighted eigenpair problem of $\Delta_{p}$, there is a corresponding $p$-Henon equation

$$
-\Delta_{p} u+|x-\overrightarrow{1}|^{r}|u|^{q} u=0, x \in \Omega,\left.\quad u\right|_{\partial \Omega}=0 .
$$


Usually, $q>p-2$. By the numerical results in [35], the symmetry of the ground state will be broken when $r$ becomes large. This interesting case is called a symmetrybreaking phenomenon. But by our numerical results in this paper, it seems that for the weighted eigenpair problems, such a phenomenon never occurs. This observation motivates us to do further numerical investigations. Our findings will be reported in [37].

5. Some theoretical issues. The original LMM method was designed for finding multiple unconstrained saddle points. In contrast, our model problem in this paper is constrained and the critical points of the Rayleigh quotient $J$ are always degenerate, which causes some theoretical issues related to the modified LMM. In this section, we present some mathematical analysis on those issues.

5.1. On convergence of the algorithm. To establish some convergence results, as suggested in Remark 2.2, we need to generalize the notion of a peak selection as follows.

Definition 5.1. A set-valued mapping $P: S_{L^{\prime}} \rightarrow 2^{B}$ is the $L-\perp$ mapping of $J$ if $\forall v \in S_{L^{\prime}}, P(v)=\left\{u=\sum_{i=1}^{n-1} t_{i} u_{i}+t_{n} v \mid \sum_{i=1}^{n} t_{i}^{2}=1,\left\langle\nabla J(u), u_{i}\right\rangle=0, i=\right.$ $1, \ldots, n-1\}$. A single-valued mapping $p: S_{L^{\prime}} \rightarrow B$ is an $L-\perp$ selection of $J$ if $p(v) \in P(v) \forall v \in S_{L^{\prime}}$. For a given $v \in S_{L^{\prime}}$, we say that $J$ has a local $L-\perp$ selection at $v$ if there is a neighborhood $\mathcal{N}(v)$ of $v$ and $p: \mathcal{N}(v) \cap S_{L^{\prime}} \rightarrow B$ s.t. $p(u) \in P(u) \forall u \in$ $\mathcal{N}(v) \cap S_{L^{\prime}}$.

The iso-homogeneous condition implies $\langle\nabla J(p(v)), p(v)\rangle=0$ and $\langle\nabla J(p(v)), v\rangle=$ 0 if $p(v) \notin L$. Then, a min-orthogonal algorithm can be designed through replacing a peak selection in the LMM approach by an $L$ - $\perp$ selection $p(v)=t_{1} u_{1}+\cdots+t_{n-1} u_{n-1}+$ $t_{n} v$, which is solved from

$$
\left\langle\nabla J\left(t_{1} u_{1}+\cdots+t_{n-1} u_{n-1}+t_{n} v\right), u_{i}\right\rangle=0, \quad i=1, . ., n-1, \sum_{i=1}^{n} t_{i}^{2}=1 .
$$

We need the following condition.

Definition 5.2. Given $u_{i} \in B$ with $\left\|u_{i}\right\|=1, i=1, \ldots, n-1$. A functional $J \in C^{1}(B, \mathbb{R})$ is said to satisfy the Palais-Smale $(P S)$ condition if any sequence $\left\{w_{i}\right\} \subset B$ of the form $w_{i}=\sum_{j=1}^{n-1} t_{j}^{i} u_{j}+t_{n}^{i} v_{i}$ for some $\left\{v_{i}\right\} \subset B,\left\|v_{i}\right\|=1$, and $\sum_{j=1}^{n}\left(t_{j}^{i}\right)^{2}=1$ such that $J\left(w_{i}\right)$ is bounded and $\nabla J\left(w_{i}\right) \rightarrow 0$, possesses a convergent subsequence.

Then with some modifications along the lines of the proofs, similar convergence results as in [36] can be established. Since the proofs are similar and very long, we omit them here.

5.2. On smoothness of $\boldsymbol{L}$ - $\perp$ selection. Since the selection of an $L$ - $\perp$ is implicitly defined and its continuity or smoothness is important for our algorithmdesign/convergence-analysis, the following implicit function theorem (IFT) approach is used to check whether $p$ is smooth. This is one of the theoretical reasons why a peak selection has to be generalized to an $L$ - $\perp$ selection. According to the definition of an $L-\perp$ selection, when $L=\left[u_{1}, u_{2}, \ldots, u_{n-1}\right], p(v)=\sum_{i=1}^{n-1} t_{i} u_{i}+t_{n} v$, where $\sum_{i=1}^{n} t_{i}^{2}=1$ and $v \in S_{L^{\prime}}$, is solved from (5.1). To apply the IFT to (5.1), we need to resolve the problem caused by the normalization $\sum_{i=1}^{n} t_{i}^{2}=1$, which represents the constraint of the problem and prevents us from taking derivative w.r.t. $t_{i}$. Since the system in (5.1) is iso-homogeneous, the normalization $\sum_{i=1}^{n} t_{i}^{2}=1$ can always be satisfied afterward through dividing each $t_{i}$ by $\left(\sum_{j=1}^{n} t_{j}^{2}\right)^{\frac{1}{2}}$. Thus the normalization 
condition can be released. Then for given $v \in S_{L^{\prime}}$, the system (5.1) contains $n$ unknowns $t_{1}, \ldots, t_{n}$ but $n-1$ equations. To obtain a square Hessian matrix of (5.1) and keep in mind the nondegeneracy condition dis $(p(v), L)>0$ in our local minimax characterization, Theorem 2.6, let us solve

$$
\left\langle\nabla J\left(t_{1} u_{1}+\cdots+t_{n-1} u_{n-1}+v\right), u_{i}\right\rangle=0, \quad i=1, \ldots, n-1 .
$$

That is, we force $t_{n}=1$ in (5.1). The IFT states that if $u=p(v)=t_{1} u_{1}+\cdots+$ $t_{n-1} u_{n-1}+v$ satisfies $(5.2)$, the $(n-1) \times(n-1)$ matrix

$$
Q=\left[\begin{array}{ccc}
\left\langle J^{\prime \prime}(u) u_{1}, u_{1}\right\rangle & \cdots & \left\langle J^{\prime \prime}(u) u_{n-1}, u_{n-1}\right\rangle \\
\cdots & \cdots & \\
\left\langle J^{\prime \prime}(u) u_{1}, u_{n-1}\right\rangle & \cdots & \left\langle J^{\prime \prime}(u) u_{n-1}, u_{n-1}\right\rangle
\end{array}\right]
$$

is invertible; i.e., $|Q| \neq 0$; then $\left(t_{1}(w), \ldots, t_{n-1}(w)\right)$ can be solved from (5.2) around $v$; i.e., $p(w)=\sum_{i=1}^{n-1} t_{i}(w) u_{i}+w$ satisfies $(5.2)$ and $\left(t_{1}(w), \ldots, t_{n-1}(w)\right)$ is continuously differentiable around $v$. Thus the $L-\perp$ selection $p$ is well defined and continuously differentiable near $v \in S_{L^{\prime}}$. Then we can normalize $p(w)$ through a differentiable operation, i.e., multiplying $p(w)$ by $t_{n}(w)=1 /\left(\sum_{i=1}^{n-1} t_{i}(w)^{2}+1\right)^{\frac{1}{2}}$ to get the new $p(w) \in[L, w]_{S} \forall w$ near $v$ in $S_{L^{\prime}}$. Then (5.1) is satisfied, and the condition $|Q| \neq 0$ can be easily numerically checked.

5.3. On an instability order of eigenfunctions. In this subsection, we establish an instability order for eigenpairs of (1.10) computed by the modified LMM. Note that the role played by the iso-homogeneous condition (1.12) to define this order is double-edged, it enables the eigenvalues of (1.10) to coincide with the critical values of the Rayleigh quotient $J$ (see Lemma 2.1), and it also makes $J$ degenerate.

Let $B$ be a Hilbert space and $J \in C^{2}(B, \mathbb{R})$. If $u^{*} \in B$ is a nondegenerate critical point of $J$, then $B=B^{-} \oplus B^{+}$, where $B^{-}$and $B^{+}$are, respectively, the maximum negative and positive definite subspaces of $J^{\prime \prime}\left(u^{*}\right)$. The integer $\operatorname{MI}\left(u^{*}\right)=\operatorname{dim}\left(B^{-}\right)$ is called the Morse index (MI) of $u^{*}$. MI is commonly used to define an instability order for nondegenerate and unconstrained saddle points in a Hilbert space [30, 41]. But MI cannot handle degenerate cases and is not defined in a Banach space. Thus another approach allowing a Banach space setting, constrained, and degenerate cases has to be developed.

In computational chemistry and physics, the following notion of saddle order is of interest.

Definition 5.3. Let $J \in C^{1}(B, \mathbb{R})$, and $u^{*} \in B$ be a critical point of $J$. If $B=B^{I} \oplus B^{D}$ for some subspaces $B^{I}, B^{D}$ in $B$ and for each $u_{1} \in B^{I}$ and $u_{2} \in B^{D}$ with $\left\|u_{1}\right\|=1$ and $\left\|u_{2}\right\|=1$, there exist constants $r_{1}>0$ and $r_{2}>0$ s.t.

$$
\begin{array}{ll}
J\left(u^{*}+t u_{1}\right) \geq J\left(u^{*}\right) & \forall 0<|t| \leq r_{1}, \\
J\left(u^{*}+t u_{2}\right)<J\left(u^{*}\right) & \forall 0<|t| \leq r_{2} .
\end{array}
$$

The integer $\operatorname{dim}\left(B^{D}\right)$ is the dimension of the maximum subspace of decreasing directions of $J$ at a critical point $u^{*}$ (a local instability index) and is called the saddle order of $u^{*}$.

Since (5.3) and (5.4) lack characterization and robustness, it is difficult for us to apply, in particular, in a Banach space. Thus in the definition, we replace (5.4) by

$$
J\left(u^{*}+t u_{2}+o(t)\right)<J\left(u^{*}\right) \quad \forall 0<|t| \leq r_{2},
$$


where $o(t)$ represents a higher order term. The integer $\operatorname{dim}\left(B^{D}\right)$ is then called the local saddle index (LSI) and will be used to define an instability order for critical points of $J$.

If $u^{*}$ is a nondegenerate saddle point of $J$ on a Hilbert space $B=B^{-} \oplus B^{+}$, then

$$
\begin{aligned}
& J\left(u^{*}+t u_{1}+o(t)\right)-J\left(u^{*}\right)=\frac{t^{2}}{2}\left\langle J^{\prime \prime}\left(u^{*}\right) u_{1}, u_{1}\right\rangle+o\left(t^{2}\right)>0, \\
& J\left(u^{*}+t u_{2}+o(t)\right)-J\left(u^{*}\right)=\frac{t^{2}}{2}\left\langle J^{\prime \prime}\left(u^{*}\right) u_{2}, u_{2}\right\rangle+o\left(t^{2}\right)<0
\end{aligned}
$$

for any $u_{1} \in B^{+}, u_{2} \in B^{-}$and $|t|$ small; i.e., $\operatorname{LSI}\left(u^{*}\right)=\operatorname{MI}\left(u^{*}\right)$. Since the definition of LSI by (5.3) and (5.5) does not concern the degeneracy of $u^{*}$, LSI is more general than MI. Next we assume that (5.3) and (5.5) are satisfied and prove that for a saddle point $u^{*}=p\left(v^{*}\right)$ found by our modified LMM, LSI $=\operatorname{dim}(L)=k$, where $L=\left[u_{1}, \ldots, u_{k}\right]$ and $u_{1}, \ldots, u_{k}$ are linear independent and normalized. Due to the iso-homogeneous condition, the definition of the Rayleigh quotient $J$, and the condition $u^{*} \notin L$, as pointed out in section 5.2, our LMM characterization

$$
v^{*}=\arg \min _{v \in S_{L^{\prime}}} \max _{u \in\left[u_{1}, \ldots, u_{k}, v\right]_{S}} J(u),
$$

i.e., $p(v)=\sum_{i=1}^{n-1} t_{i} u_{i}+t_{n} v, \sum_{i=1}^{n} t_{i}=1$, is equivalent to solving

$$
\bar{v}=\arg \min _{v \in S_{L^{\prime}}} \max _{\left(t_{1}, \ldots, t_{k}\right) \in \mathbb{R}^{k}} J\left(t_{1} u_{1}+\cdots+t_{k} u_{k}+v\right),
$$

i.e., $p(v)=t_{1} u_{1}+\cdots+t_{k} u_{k}+v$. However, (5.7) has an advantage that the local maximization at the second level is taken on a finite-dimensional space, not just on a manifold as in (5.6).

Theorem 5.4. Let $B$ be a Banach space s.t. $\|\cdot\|^{\prime}$ exists on $B \backslash\{0\}$ and $v^{*} \in S_{L^{\prime}}$. Assume that $J$ has a local peak selection $p$ w.r.t. $L$ at $v^{*}$ s.t. $p$ is differentiable at $v^{*}, u^{*} \equiv p\left(v^{*}\right) \notin L$, and $v^{*}=\arg \min _{v \in S_{L^{\prime}}} J(p(v))$. If we assume that the local maximization in (5.7) is strict, then $u^{*}$ is a saddle point of LSI $k=\operatorname{dim}(L)$.

Proof. Let $B^{D}$ and $B^{I}$ be defined as in (5.3) and (5.5). If $k=0$, there is no local maximization in (5.7), or, $v^{*}=p\left(v^{*}\right)$ is a local minimum point of $J$. It is clear that $k=0=\operatorname{dim}\left(B^{D}\right)$. Now we assume that $k>0$. Since $k>\operatorname{dim}\left(B^{D}\right)$ implies $L \cap B^{I} \neq \emptyset$, it contradicts our assumption that the local maximum in (5.7) is strict. Thus we have $k \leq \operatorname{dim}\left(B^{D}\right)$. To prove $k=\operatorname{dim}\left(B^{D}\right)$, recall $B=L \oplus L^{\prime}, v^{*} \in S_{L^{\prime}}$. For each $v \in L^{\prime}$ with $v \neq 0$, we define $E(v)=\frac{v}{\|v\|}$. We have $E^{\prime}(v) w=\frac{w}{\|v\|}-\frac{v}{\|v\|}\left\langle\left\|\frac{v}{\|v\|}\right\|^{\prime}, w\right\rangle$. Let $L_{v^{*}}^{\perp}=\left\{w \in L^{\prime}:\left\langle\left\|v^{*}\right\|^{\prime}, w\right\rangle=0\right\}$. For each $w \in L^{\prime}$, we can write $w=c v^{*}+\left(w-c v^{*}\right)$, where $c=\left.\frac{d}{d \alpha}\right|_{\alpha=0}\left\|v^{*}+\alpha w\right\|=\left\langle\left\|v^{*}\right\|^{\prime}, w\right\rangle$ and $\left\langle\left\|v^{*}\right\|^{\prime}, v^{*}\right\rangle=\left\|v^{*}\right\|=1$. Thus $w-c v^{*} \in$ $L_{v^{*}}^{\perp}$. Since $\left[v^{*}\right] \cap L_{v^{*}}^{\perp}=\{0\}$, we have $L_{v^{*}}^{\perp} \oplus\left[v^{*}\right]=L^{\prime}$. For each $w \neq 0$ in $L_{v^{*}}^{\perp}$, $E^{\prime}\left(v^{*}\right) w=w$. Let $\alpha(s) \equiv \mathcal{P}\left(p\left(E\left(v^{*}+s w\right)\right)\right)=E\left(v^{*}+s w\right)$, where $\mathcal{P}$ is the linear projection operator onto $L^{\prime}$. We have $\alpha^{\prime}(0)=\mathcal{P}\left(p^{\prime}\left(E\left(v^{*}\right)\right) E^{\prime}\left(v^{*}\right) w\right)=E^{\prime}\left(v^{*}\right) w=w$, i.e., $\mathcal{P}\left(p^{\prime}\left(v^{*}\right) w\right)=w$. It follows that $w \in\left\{p^{\prime}\left(v^{*}\right)(w), L\right\}$. Since $w \in L_{v^{*}}^{\perp} \subset L^{\prime}$, we have $p^{\prime}\left(v^{*}\right) w \notin L$. This leads to

$$
p^{\prime}\left(v^{*}\right)\left(L_{v^{*}}^{\perp}\right) \oplus\left[v^{*}\right] \oplus L=B .
$$

Now we suppose $k<\operatorname{dim}\left(B^{D}\right)$. Then (5.8) implies $\left(p^{\prime}\left(v^{*}\right)\left(L_{v^{*}}^{\perp}\right) \oplus\left[v^{*}\right]\right) \cap B^{D} \neq\{0\}$. Since $J(v) \equiv J(t v) \forall t \neq 0$ and $\forall v \neq 0$, we must have $p^{\prime}\left(v^{*}\right)\left(L_{v^{*}}^{\perp} \cap B^{D} \neq\{0\}\right.$. Let 
$w \in L_{v^{*}}^{\perp}$ s.t. $p^{\prime}\left(v^{*}\right)(w) \in\left(B^{D} \backslash\{0\}\right)$. Then $E\left(v^{*}+s w\right) \in \mathcal{N}\left(v^{*}\right) \cap S_{L^{\prime}}$ for $|s|$ small. It follows that

$$
\begin{aligned}
p\left(E\left(v^{*}+s w\right)\right) & =p\left(E\left(v^{*}\right)\right)+s p^{\prime}\left(E\left(v^{*}\right)\right) E^{\prime}\left(v^{*}\right)(w)+o(s) \\
& =p\left(v^{*}\right)+s p^{\prime}\left(v^{*}\right)(w)+o(s)=u^{*}+s p^{\prime}\left(v^{*}\right)(w)+o(s) .
\end{aligned}
$$

Since $p^{\prime}\left(v^{*}\right)(w) \in\left(B^{D} \backslash\{0\}\right)$, we have, for $|s|$ small,

$$
J\left(p\left(v^{*}(s)\right)\right)=J\left(u^{*}+s p^{\prime}\left(v^{*}\right)(w)+o(s)\right)<J\left(u^{*}\right)=J\left(p\left(v^{*}\right)\right) .
$$

On the other hand, by our assumption on $v^{*}$ and (5.7), we should have

$$
J\left(p\left(v^{*}(s)\right)\right)=\max _{\left(t_{1}, \ldots, t_{k}\right) \in \mathbb{R}^{k}} J\left(t_{1} u_{1}+\cdots+t_{k} u_{k}+v^{*}(s)\right) \geq J\left(p\left(v^{*}\right)\right) .
$$

This leads to a contradiction. We conclude that $k=\operatorname{dim}\left(B^{D}\right)$.

The integer $k=\operatorname{dim}(L)$ is called the local minimax index of the constrained saddle point $u^{*}=p\left(v^{*}\right)$ of $J$. Theorem 5.4 states that LSI $=$ LMI. Following our modified LMM, we start with $k=0$ or $L=\{0\}$, to find a local minimum point $u^{1}$ of $J$. The LMI of $u_{1}$ is $k=0$. Then we go to $k=1$ or $L=\left[u_{1}\right]$ to find a saddle point $u_{2}$ of $J$ of LMI $k=1, \ldots$ Continuing in this way, we use $L=\left[u_{1}, \ldots, u_{k}\right]$ to find a saddle point $u_{k+1}$ of $J$ of LMI $=k$. Therefore for each $k=0,1, \ldots$, at least one saddle point $u_{k+1}$ with LMI $=k$ will be found. The dimension of the maximum subspace of decreasing directions of $J$ (energy function) at $u_{k+1}$ is LSI $=\mathrm{LMI}=k$. Thus the order of LMI coincides with the instability order of saddle points. In contrast, the order of the LMI can be easily followed by our modified LMM method. It is clear that $J\left(u_{j}\right)<J\left(u_{k}\right)$ for $j=0,1, \ldots, k-1$. Since Theorem 2.6 states $J\left(u_{k}\right)=\lambda_{k}$, we also have $\lambda_{j}<\lambda_{k}$ for $j=0,1, \ldots, k-1$. Since the modified LMM is local, this order may be partial and contains multiple branches. If the order contains only one branch, then no eigenfunction will be missed and the order of eigenfunctions coincides with the natural order of their eigenvalues. However, when the order contains multiple branches, i.e., more than one eigenfunction has the same LMI or a bifurcation takes place, each branch contains a sequence of eigenfunctions according to their LMI whose order coincides with the natural order of their eigenvalues. For linear eigenpair problems as in Example 1.1, all eigenfunctions with the same LMI have the same eigenvalues. For a nonlinear eigenpair problem, as we found in our numerical results, a significant difference is that eigenfunctions with the same LMI may have different eigenvalues. Eigenvalues of two eigenfunctions from two different branches cannot be compared, whether they have the same or different LMI.

Acknowledgment. The authors thank two anonymous referees for their helpful comments.

\section{REFERENCES}

[1] E. Cances, H. Galicher and M. Lewin, Computing electronic structures: A new multiconfiguration approach for excited states, J. Comput. Phys., 212 (2006), pp. 73-98.

[2] Y. S. Choi And P. J. McKenna, A mountain pass method for the numerical solution of semilinear elliptic problems, Nonlinear Anal., 20 (1993), pp. 417-437.

[3] M. Cuesta, Eigenvalue problems for the p-Laplacian with indefinite weights, Electronic J. Differential Equations, 2001 (2001), pp. 1-9.

[4] J. I. Diaz, Nonlinear Partial Differential Equations and Free Boundaries, Vol. I, Elliptic Equations, Res. Notes Math. 106, Pitman, Boston, 1985. 
[5] Z. Ding, D. Costa, ANd G. Chen, A high linking algorithm for sign-changing solutions of semilinear elliptic equations, Nonlinear Anal., 38 (1999), pp. 151-172.

[6] R. J. DodD, Approximate solutions of the nonlinear Schrödinger equation for ground and excited states of Bose-Einstein condensates, J. Res. Natl. Inst. Stand. Technol., 545 (1996), pp. 1-8.

[7] P. Drabek And S. B. Robinson, Resonance Problems for the p-Laplacian, J. Funct. Anal., 169 (1999), pp. 189-200.

[8] J. J. García-RIPOll and V. M. PÉrez-García, Optimizing Schrödinger functionals using Sobolev gradients: Applications to quantum mechanics and nonlinear optics, SIAM J. Sci. Comput., 23 (2001), pp. 1316-1334.

[9] J. J. Garcia-Ripoll and V. M. Perez-Garcia, Stable and unstable vortices in multicomponent Bose-Einstein condensates, Phys. Rev. Lett., 84 (2000), pp. 4264-4267.

[10] E. P. Gross, Structure of a quantized vortex in Boson systems, Nuovo Cimento, 20 (1961), pp. 454-477.

[11] G. Henkelman, G. Johannesson, and H. Jonsson, Methods for finding saddle points and minimum energy paths, in Theoretical Chemistry and Physics, D. Schwartz, ed., vol. 5, Kluwer Academic Publishers, Dordrecht, The Netherlands, 2000, pp. 269-300.

[12] http://www.ams.org/msnmain?fn=130\&pg1=CC\&op1=AND\&s1=35B38\&fmt=hl

[13] http://www.ams.org/msnmain?fn=130\&pg1=CC\&op1=AND\&s1=58E05\&fmt=hl

[14] http://www.ams.org/msnmain?fn=130\&pg1=CC\&op1=AND\&s1=35Q55\&fmt=hl

[15] S. V. IzBAsh, O Filtracii V Kropnozernstom Materiale, Izv. Nauchnoissled, Inst. Gidrotechniki (NIIG), Leningrad, USSR, 1931.

[16] C. E. Kenig, The Cauchy Problems for the Quasilinear Schrodinger Equations, Lecture notes to be published by American Mathematical Society.

[17] S. Komineas and N. PapanicolaOu, Static solitary waves in axisymmetric Bose-Einstein condensates, Laser Phys., 14 (2004), pp. 571-574.

[18] P. Lindqvist, On a Nonlinear Eigenvalue Problem, Fall School in Analysis Lectures, Ber. Univ. Jyväskylä Math. Inst., 68 (1991), pp. 33-54.

[19] L. Lefton AND D. Wei, Numerical approximation of the first eigenpair of the p-Laplacian using finite elements and the penalty method, Numer. Funct. Anal. Optim., 18 (1997), pp. 389-399.

[20] Y. Li AND J. Zhou, A minimax method for finding multiple critical points and its applications to semilinear PDEs, SIAM J. Sci. Comput., 23 (2001), pp. 840-865.

[21] Y. Li AND J. ZHOU, Convergence results of a local minimax method for finding multiple critical points, SIAM J. Sci. Comput., 24 (2002), pp. 865-885.

[22] J.-Q. LiU, Y.-Q. WANG, AND Z.-Q. WANG, Soliton solutions for quasilinear Schrodinger equation, II, J. Differential Equations, 187 (2003), pp. 473-493.

[23] S. LiU, Existence of solutions to a superlinear p-Laplacian equation, Electronic J. Differential Equations, 2001 (2001), pp. 1-6.

[24] E. Montefusco And V. Radulescu, Nonlinear eigenvalue problems for quasilinear operators on unbounded domains, Nonlinear Differential Equations Appl., 8 (2001), pp. 481-497.

[25] Z. H. Musslimani, M. Segev, D. N. Christodoulides, and M. Soljacic, Composite multihump vector solitons carrying topological charge, Phy. Rev. Lett., 84 (2000), pp. 1164-1167.

[26] J. M. Neuberger And J. Swift, Newton's method and Morse index for semilinear elliptic PDEs, Int. J. Bifur. Chaos, 11 (2001), pp. 802-820.

[27] L. P. Pitaevskit, Vortex lines in an imperfect Bose gas, Soviet Phys. JETP, 13 (1961), pp. 451-454.

[28] J. Schulz, M. Tchaplyguine, T. Rander, O. Burneholm, S. Svensson, R. Sankari, S. Heinsmki, H. Aksela, S. Aksela, And E. KukK, Shakedown in core photoelectron spectra from aligned laser-excited Na atoms, Phys. Rev. A, 72 (2005), 010702.

[29] J. Schulz, M. Tchaplyguine, T. Rander, H. Bergersen, A. Lindblad, G. hrwall, S. Svensson, S. Heinsmki, R. Sankari, S. Osmekhin, S. Aksela, and H. Aksela, Final state selection in the $4 p$ photoemission of Rb by combining laser spectroscopy with soft-xray photoionization, Phys. Rev. A, 72 (2005), 032718.

[30] J. Smoller, Shock Waves and Reaction-Diffusion Equation, Springer-Verlag, New York, 1983.

[31] S. I. Themelis, P. Lambropoulos, and F. J. Wuilleumier, Laser-induced transitions between core excited states of Na, J. Phys. B, 38 (2005), pp. 2119-2132.

[32] Z.-Q. Wang And J. Zhou, A local minimax-Newton method for finding multiple saddle points with symmetries, SIAM J. Numer. Anal., 42 (2004), pp. 1745-1759.

[33] Z.-Q. WANG AND J. Zhou, An efficient and stable method for computing multiple saddle points with symmetries, SIAM J. Numer. Anal., 43 (2005), pp. 891-907.

[34] Z. XIE, C. Chen, AND Y. Xu, An improved search-extension method for computing multiple solutions of semilinear PDEs, IMA J. Numer. Anal., 25 (2005), pp. 549-576. 
[35] X. Yao AND J. Zhou, A minimax method for finding multiple critical points in Banach spaces and its application to quasi-linear elliptic PDE, SIAM J. Sci. Comput., 26 (2005), pp. 1796-1809.

[36] X. YAO AND J. ZHOU, Unified convergence results on a minimax algorithm for finding multiple critical points in Banach spaces, SIAM J. Numer. Anal., to appear.

[37] X. YaO AND J. Zhou, Some Numerical Investigation of the p-Henon Equation, preprint.

[38] X. Yao and J. Zhou, Numerical Methods for Computing Nonlinear Eigenpairs: Part II. Non Iso-Homogeneous Cases, submitted.

[39] E. ZeIdLer, Nonlinear Functional Analysis and Its Applications III, Springer-Verlag, New York, 1985.

[40] J. ZHOU, A local mini-orthogonal method for finding multiple saddle points, J. Math Anal. Appl., 291 (2004), pp. 66-81.

[41] J. ZHOU, Instability analysis of saddle points by a local minimax method, Math. Comp., 74 (2005), pp. 1391-1411. 
Copyright of SIAM Journal on Scientific Computing is the property of Society for Industrial and Applied Mathematics and its content may not be copied or emailed to multiple sites or posted to a listserv without the copyright holder's express written permission. However, users may print. download, or email articles for individual use. 
Copyright of SIAM Journal on Scientific Computing is the property of Society for Industrial and Applied Mathematics and its content may not be copied or emailed to multiple sites or posted to a listserv without the copyright holder's express written permission. However, users may print. download, or email articles for individual use. 